Kardiologe $2022 \cdot 16: 38-45$

https://doi.org/10.1007/s12181-021-00523-3

Angenommen: 26. November 2021

Online publiziert: 13. Januar 2022

(c) Der/die Autor(en) 2022

\section{Moderne Therapieoptionen der Trikuspidalklappeninsuffizienz}

\section{Von den leitliniengerechten chirurgischen Reparatur- und interventionellen Segeltherapiestrategien zum interventionellen Trikuspidalklappenersatz}

\author{
Michaela M. Hell' · Hendrik Treede ${ }^{2}$ - Thomas Münzel' · \\ Ralph Stephan von Bardeleben ${ }^{1}$ \\ 'Zentrum für Kardiologie, Kardiologie 1, Universitätsmedizin Mainz, Mainz, Deutschland \\ ${ }^{2}$ Klinik und Poliklinik für Herz- und Gefäßchirurgie, Universitätsmedizin Mainz, Mainz, Deutschland
}

\title{
Zusammenfassung
}

Das fehlende Bewusstsein für die klinische Relevanz der Trikuspidalklappeninsuffizienz (TI) sowie die hohe intrahospitale Mortalität führten bis vor Kurzem zu einer späten Patientenvorstellung mit häufig bereits irreversibler rechtsventrikulärer Schädigung. Innovative Therapietechniken, neue Bildgebungstechniken und das bessere hämodynamische Verständnis des rechten Herzens revolutionierten in den letzten Jahren die Behandlungsmöglichkeiten der TI. Die vorliegende Arbeit diskutiert die Bedeutung der Rechtsherzfunktion für die Trikuspidalklappentherapie und stellt moderne interventionelle und minimal-invasive chirurgische Techniken vor. Zudem gibt sie einen Ausblick über die aktuelle Entwicklung der innovativen TranskatheterTrikuspidalklappenersatz-Systeme.

\section{Schlüsselwörter}

Transkatheter-Herzklappentherapie - Minimal-invasive Chirurgie · Rechtsventrikuläre Funktion · Intrahospitale Mortalität · Hämodynamik

Die Trikuspidalklappeninsuffizienz (TI) ist schweregradabhängig ein Prädiktor für schlechte klinische Verläufe und erhöhte Mortalität. Das fehlende Bewusstsein für die klinische Relevanz dieses Vitiums sowie die hohe perioperative Mortalität führten bis vor Kurzem zu einer späten Patientenvorstellung mit bereits irreversibler rechtsventrikulärer (RV) Schädigung. Innovative Therapien mittels Transkatheter- und minimal-invasiver chirurgischer Technik, neue Bildgebungstechniken und das bessere hämodynamische Verständnis des rechten Herzens mit einer frühzeitigen Patientenvorstellung revolutionierten in den letzten Jahren die Behandlungsmöglichkeiten bei TI.

Die TI ist die zweithäufigste Herzklappenerkrankung. Bei ca. $85 \%$ der Patienten liegt eine funktionelle (oder sekundäre) TI vor, bei der es durch eine Dilatation der rechtsseitigen Herzhöhlen und des Triku- spidalklappenringes zu einer Beeinträchtigung der Segelkoaptation kommt. Sie ist in mehr als der Hälfte der Fälle eine Folge von Linksherzerkrankungen inklusive der Aorten- und Mitralklappenerkrankungen und in ca. $15 \%$ einer pulmonalen Hypertonie (PH). Nur in seltenen Fällen liegt eine primäre TI vor. Die Rolle der ventrikulären Schrittmacher- und ICD(implantierbarer Kardioverter/Defibrillator)-Sonden wird derzeit neu und umfassender bewertet.

\section{Eine komplexe Klappe}

Ein Verständnis für die komplexe Trikuspidalklappenanatomie und die pathophysiologischen Veränderungen bei einer TI ist für die Diagnostik und Auswahl der optimalen Therapiestrategie unabdingbar. Die Trikuspidalklappe (TK) hat einen asymmetrischen sattelförmigen fibrösen Anulus, 3 Segel (anterior, posterior und septal) 
und einen subvalvulären Apparat, bestehend aus den Chordae tendinae und den Papillarmuskeln.

Bei einer funktionellen TI kommt es zu signifikanten Veränderungen in der Geometrie des TK-Apparates - das sind v. a. eine Abflachung und Dilatation des Anulus sowie ein "Tethering" der Segel (vermehrte Zugspannung an den Klappensegeln aufgrund des Chordaezuges bei RV-Dilatation).

Die TK bildet eine funktionelle Einheit mit den rechten Herzhöhlen. Die spezielle Architektur des rechten Ventrikels mit den prominenten Trabekeln, Papillarmuskeln und dem Moderatorband und deren mögliche Interaktion mit einem TranskatheterImplantationssystem müssen berücksichtigt werden.

Bei Eingriffen an der TK ist außerdem die enge anatomische Beziehung zur rechten Koronararterie (RCA, Verlauf entlang des anterioren und posterioren Anulus) und zu dem AV(atrioventrikular)-Knoten im KochDreieck (begrenzt posterior durch die Einmündung des Sinus coronarius, anterior durch den Rand des septalen TK-Ringes und superior durch die Todaro-Sehne [Verlängerung der Eustachi-Klappe zum Trigonum fibrosum dextrum]) zu berücksichtigen.

\section{Abkürzungen}

\begin{tabular}{|c|c|}
\hline$A V$ & Atrioventrikular \\
\hline$C T$ & Computertomographie \\
\hline$E R O A$ & $\begin{array}{l}\text { Effektive Regurgitationsöffnungsflä- } \\
\text { che }\end{array}$ \\
\hline$F$ & French \\
\hline$H L M$ & Herz-Lungen-Maschine \\
\hline$I C D$ & $\begin{array}{l}\text { Implantierbarer Kardioverter/ } \\
\text { Defibrillator }\end{array}$ \\
\hline$I C R$ & Interkostalraum \\
\hline MRT & Magnetresonanztomographie \\
\hline $\mathrm{PH}$ & Pulmonale Hypertonie \\
\hline$R C A$ & Rechte Koronararterie \\
\hline RV & Rechter Ventrikel/rechtsventrikulär \\
\hline TAVI & $\begin{array}{l}\text { Transcatheter aortic valve implanta- } \\
\text { tion }\end{array}$ \\
\hline TEE & $\begin{array}{l}\text { Transösophageale Echokardiogra- } \\
\text { phie }\end{array}$ \\
\hline TEER & Transcatheter edge-to-edge repair \\
\hline $\mathrm{TI}$ & Trikuspidalklappeninsuffizienz \\
\hline$T K$ & Trikuspidalklappe \\
\hline$V C$ & Vena contracta \\
\hline
\end{tabular}

\section{Diagnostik}

Die Echokardiographie ist die zentrale Bildgebungsmodalität bei der TI. Zur Schweregradbewertung der TI wurde als Erweiterung des 3-Stufen-Modells (gering-, mittel- und hochgradig) ein 5-Stufen-Modell vorgeschlagen (gering-, mittel-, hochgradig, massiv und „torrential“ (sintflutartig oder reißend)) [3]. Die Beurteilung der Segelmorphologie, die Vermessung des Koaptationsdefektes und die Lokalisation des Hauptinsuffizienzjets mittels transösophagealer Echokardiographie (TEE) in tief-ösophagealen und transgastrischen Schallfenstern sind notwendig, technisch geeignete Therapiekandidaten zu identifizieren. Ergänzend zur 2-D-TEE bieten verschiedene 3-DTEE-Techniken eine bessere Visualisierung der komplexen Anatomie (- Abb. 1a, b). Weiterhin erfolgt eine Beurteilung der rechtsseitigen Herzhöhlen, des pulmonalvaskulären Widerstands und des systolischen Pulmonalisdruckes.

Die Magnetresonanztomographie (MRT) ist der Goldstandard zur Bestimmung der RV-Volumina und Funktion, der jedoch aufgrund zu geringer Verfügbarkeit nicht routinemäßig zum Einsatz kommt. Die Computertomographie (CT) ist in der Prozedurplanung für eine TranskatheterAnuloplastie und einen TranskatheterTK-Ersatz essenzieller Teil der präinterventionellen Bildgebung [4]. Es können die Anulusebene und der Abstand zu angrenzenden Strukturen präzise vermessen, der Zugangsweg überprüft und die optimale fluoroskopische Projektionsebene für die Implantation vorhergesagt werden (• Abb. 1c). Zudem können CTDatensätze auch intraprozedural zur Fusionsbildgebung mit der Fluoroskopie oder TEE verwendet werden (• Abb. 1d). Zur besseren räumlichen Visualisierung steht eine holographische Modellgenerierung der TK aus 3-D-Echodaten zur Verfügung (- Abb. 1e, f).

\section{Therapie}

Ein isolierter operativer Eingriff bei schwergradiger $\mathrm{Tl}$ wurde in der Vergangenheit meist erst in einem späten Krankheitsstadium durchgeführt. Die intrahospitale Mortalität dieses Hochrisikoeingriffs lag mit 10\% deutlich über der eines isolierten Aorten- oder Mitralklappeneingriffes. Daher werden noch viele Patienten gar nicht für eine operative Therapieoption evaluiert, sondern medikamentös-konservativ behandelt. Die diuretische Medikation kann zunächst zwar eine subjektive Besserung der symptomatischen rechtsführenden Herzinsuffizienz erreichen, die Erkrankung aber schreitet ungehindert fort.

\section{Der richtige Zeitpunkt für die richtige Therapie}

Neben der Auswahl des Therapieverfahrens ist die rechtzeitige Behandlung in Bezug auf die RV-Funktion für das Patientenoutcome von zentraler Bedeutung. Ein geringes pathophysiologisches Verständnis der auch gerne als "forgotten valve" bezeichneten Herzklappe sowie fehlende echokardiographische Konzepte zur Aufarbeitung der hochkomplexen Klappe in der täglichen Praxis führten bis vor Kurzem zu einer späten Vorstellung der Patienten erst im Stadium der rezidivierenden stationären Dekompensation mit teilweise bereits irreversibler RV-Schädigung. Die - Abb. 2 zeigt die verschiedenen Stadien der TI in Bezug auf die RV-Funktion.

Empfehlungen zur Therapie der sekundären Trikuspidalklappeninsuffizienz nach aktuellen Leitlinien

Aufgrund der hohen Mortalität bei einem herzchirurgischen Zweiteingriff zur isolierten Behandlung einer schwergradigen $\mathrm{TI}$ wird die chirurgische Mitbehandlung der TK-Pathologie bereits bei der primären linksseitigen Herzklappenoperation in den aktuellen europäischen Leitlinien zu Herzklappenerkrankungen von 2021 favorisiert (IC/IB-Empfehlung bei schwergradiger primärer/sekundärer TI, Ila C/Ila B-Empfehlung bei mittelgradiger primärer $\mathrm{Tl} /$ leichtoder mittelgradiger sekundärer TI mit vergrößertem Anulus $>4 \mathrm{~cm}$ ) [8]. Bei Fehlen einer schweren $\mathrm{PH}$ oder RV-Dysfunktion kann durchaus ein isolierter operativer Eingriff bei schwergradiger TI auch Jahre nach erfolgter linksseitiger Herzoperation zu einer deutlichen Verbesserung der Symptomatik und Prognose führen (Ila B-Empfehlung). Erstmals wird auch für das trans- 

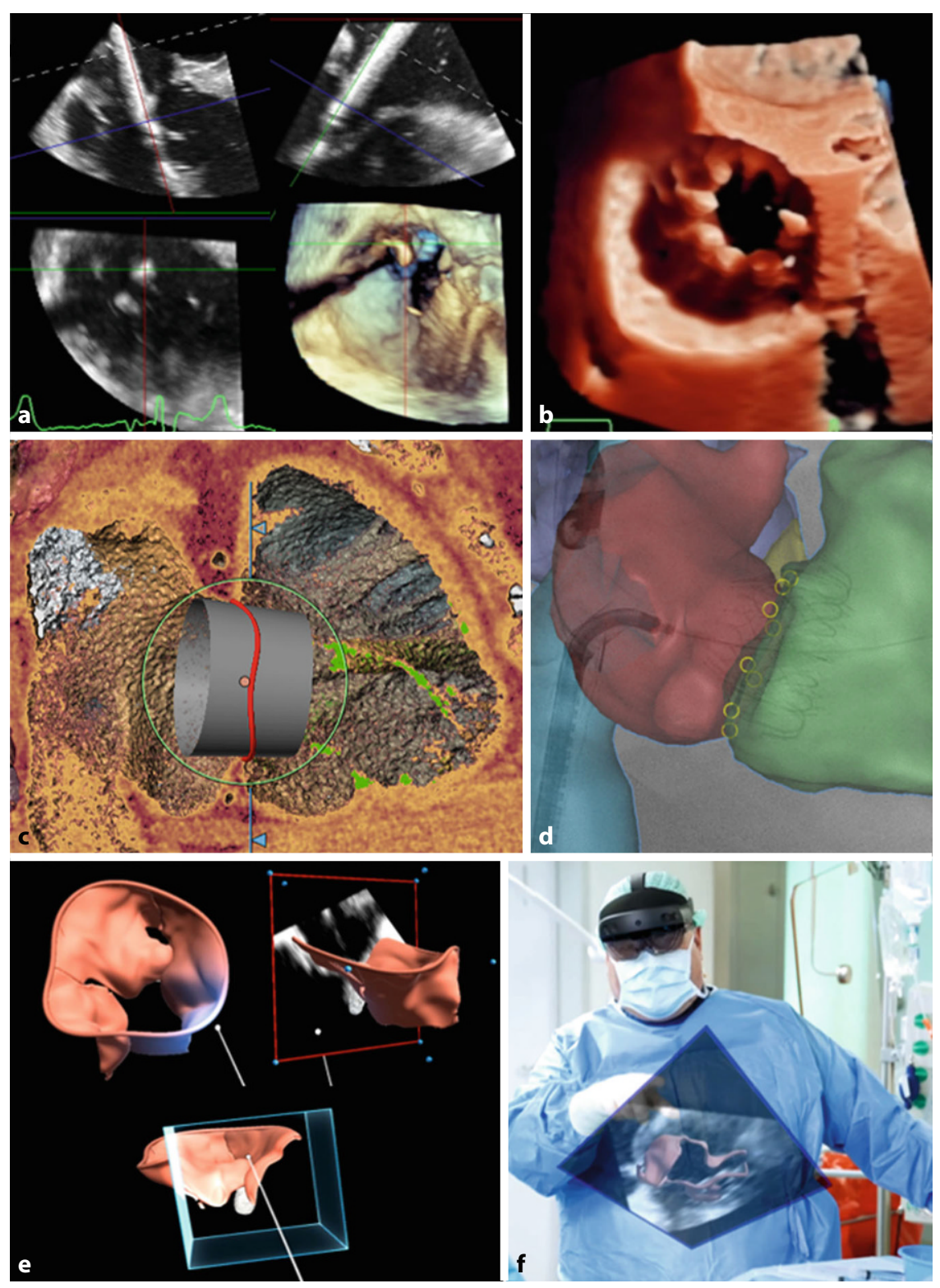

Abb. 1 \ Multimodale Bildgebungstechniken zur Diagnostik und Therapieplanung bei Trikuspidalklappeninsuffizienz (TI). a, b 3-D-TEE (transösophageale Echokardiographie) (a "real-time" multiplanare Rekonstruktion, b Transillumination). c Prothesensimulation im CT(Computertomographie)-Datensatz. d CT-Fluoroskopie-Fusionsbildgebung. e, f Holographische TK(Trikuspidalklappe)-Analyse aus 3-D-Echodaten im Hybridoperationsaal

kathetergestützte Reparaturverfahren eine Ilb C-Empfehlung ausgesprochen.

\section{Das Herzteam - Festlegung der optimalen Therapiestrategie}

Für die isolierte Behandlungsstrategie der sekundären TI existieren bisher kaum klinische Standards. Dies liegt an dem noch unzureichend verstandenen Einfluss der RV-Funktion, dem optimalen Therapiezeitpunkt, fehlenden Langzeitdaten zu neuen
Therapieverfahren und geeigneten klappenspezifischen Risikoscores.

Der fachlichen Diskussion der Befunde in einem für TI erfahrenen multidisziplinären Herzteam kommt daher eine zentrale Rolle bei der optimalen Therapiefestlegung zu. Neben den Parametern Alter, Gebrechlichkeit, Lebenserwartung, wichtige Komorbiditäten (Nierenfunktion, fixierte pulmonalarterielle Hypertonie) und Bildgebungsbefunden sollten auch der individuelle Patientenwunsch, die Langzeit- perspektive für eine optimale Lebensqualität und Optionen eines evtl. notwendigen Folgeeingriffes in Betracht gezogen werden.

Die Toolbox der verfügbaren Transkatheter- und operativen Therapiemöglichkeiten ist in - Abb. 3 zusammengefasst. - Tab. 1 gibt einen Überblick über den Anspruch an moderne chirurgische und Transkatheter-TK(Trikuspidalklappe)-Therapien.

\section{Minimal-invasive chirurgische Trikuspidalklappenrekonstruktion und -ersatz}

Die hochgradige TI stellt die Hauptindikation für einen isolierten chirurgischen Eingriff an der TK dar. Handelt es sich um eine sekundäre TI, ist meist die Möglichkeit einer Klappenrekonstruktion gegeben. Bei primärer Tl oder im seltenen Fall einer TKStenose muss dagegen auch ein bevorzugt biologischer prothetischer Klappenersatz in Betracht gezogen werden. Ziele der TKRekonstruktion sind die Wiederherstellung einer suffizienten Segelkoaptation, eine Reduktion des Anulusdiameters und eine Reduktion der RV-Nachlast. Wurden früher häufiger Nahttechniken (DeVega-Plastik, Kay-Plastik) zur Anuloplastie eingesetzt, haben sich aufgrund der besseren Langzeitdaten inzwischen Ringimplantationen mit 3-D-geformten semirigiden und rigiden Anuloplastieringen durchgesetzt. Ziel ist die Wiederherstellung der physiologischen Geometrie der Klappe. Die Ringimplantate sind offen gestaltet, um das septal gelegene Koch-Dreick mit dem darin befindlichen AV-Knoten zu schonen. Die Auswahl der korrekten Ringgröße erfolgt durch Messung der Distanz von der anteroseptalen zur posteroseptalen Kommissur.

Wurden früher mediane Sternotomien und Operationen im kardioplegischen Herzstillstand durchgeführt, so haben sich heute in erfahrenen Zentren moderne minimal-invasive Verfahren und Operationen am schlagenden Herzen durchgesetzt.

Über einen $5 \mathrm{~cm}$ Hautschnitt erfolgen zunächst eine rechtslaterale Minithorakotomie im 4. ICR (Interkostalraum) und das Einbringen einer 3-D-Endoskopiekamera (- Abb. 4a, b). Die Operation findet nach Einbringen eines Weichgeweberetraktors vollendoskopisch ohne Rippenspreizung 


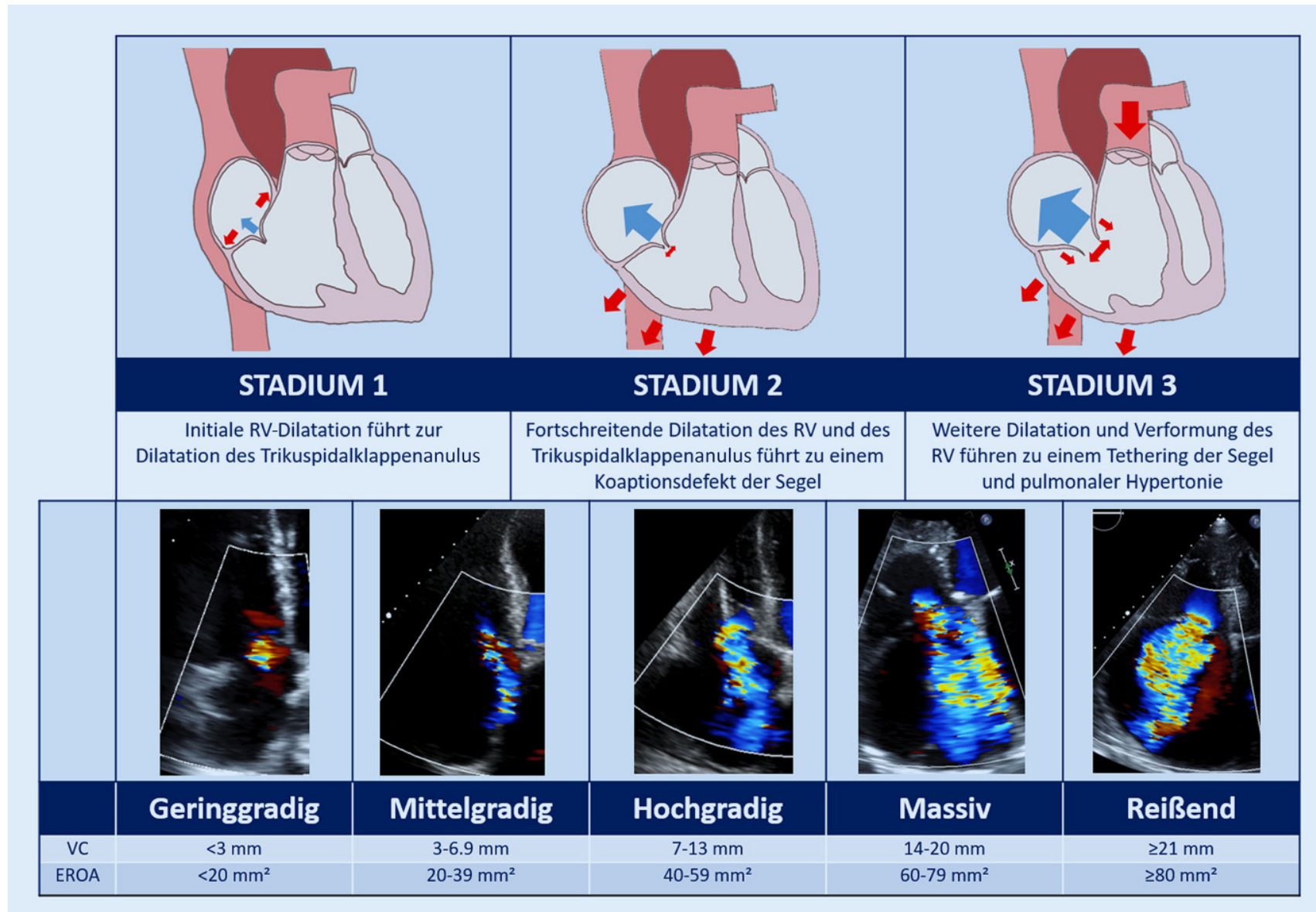

Abb. 2 \ Stadien der Trikuspidalklappeninsuffizienz (TI) in Bezug auf Rechtsherzfunktion. Die Einteilung nach Hahn et al. erfolgt nach V. contracta $(V C)$ und effektiver Regurgitationsöffnungsfläche $(E R O A)$. RV rechtsventrikulär

statt. Der Anschluss der Herz-Lungen-Maschine (HLM) erfolgt über die Kanülierung von A. und V. femoralis. Die Operation findet in Normothermie statt. Nach Anschlingen beider $\mathrm{Vv}$. cavae wird der rechte Vorhof eröffnet und mithilfe eines Klappenretraktors die TK am schlagenden Herzen endoskopisch dargestellt. Zuvor muss ein persistierendes Foramen ovale ausgeschlossen werden wegen der Gefahr der Luftembolie. Das kontinuierlich aus dem Sinus coronarius abgeleitete Blut wird über einen Sauger wieder der HLM zugeführt. Das Arbeiten am schlagenden Herzen erlaubt dabei die sehr genaue Abgrenzung des Segelgewebes vom Anulusgewebe und hilft beim korrekten Setzen der Nähte (-Abb. $\mathbf{4 c}$, d). Eventuell operationsbedingte Konduktionsstörungen durch Beeinträchtigungen des AV-Knotens können zudem gleich erkannt und verhindert werden. Führt die Implantation eines Ringimplantats in der Wasserprobe (Prüfung der Klappenschlussfähigkeit durch Applikation von Kochsalzlösung) nicht zu einer ausreichenden Segelkoaptation, z.B. im Falle eines ausgeprägten Tetherings bei sekundärer TI, kann zusätzlich eine Segelaugmentation des anterioren oder septalen Segels durch einen autologen oder xenogenen Perikardpatch erfolgen. Bei einer primären TI mit Segelprolaps oder "flail leaflet" können künstliche Sehnenfäden implantiert werden. Diese Eingriffe erfordern klappenspezifische Erfahrungen in der Längenbestimmung der Chordae, da die Volumen- und Größenvarianz des rechten Herzens hoch ist. Besteht keine Möglichkeit der Reparatur (z.B. Karzinoidsyndrom, Endokarditis), kann über denselben Zugang auch ein biologischer Klappenersatz erfolgen. Nach Entlüftung und Verschluss des rechten Vorhofs wird unter echokardiographischer Kontrolle der systolischen Funktion beider Ventrikel die
HLM zurückgefahren und bei stabiler Hämodynamik entfernt. Liegt bei chronischer Rechtsherzbelastung noch eine Instabilität vor, werden die femoral platzierten Kanülen an ein extrakorporales Membranoxygenierungsgerät angeschlossen, und ein kontrolliert prolongierter WeaningProzess wird gestartet.

\section{Transkatheterreparatur - Benefit und Limitationen}

Der häufigste Transkatheter-TK-Eingriff ist die transvenöse, transfemorale Edge-toEdge-Reparatur der Segel (,transcatheter edge-to-edge repair" [TEER]), bei dem das TriClip-Generation-3- oder -4-System mit XT(W)/NT(W) (Abbott, Chicago, IL, USA) oder Pascal/Pascal Ace Device (Edwards Lifesciences, Irvine, CA, USA) an den TKSegeln fixiert wird. Die Multicenterstudien TRILUMINATE und die randomisierte TRILUMINATE Pivotal-Studie (TriClip G3 


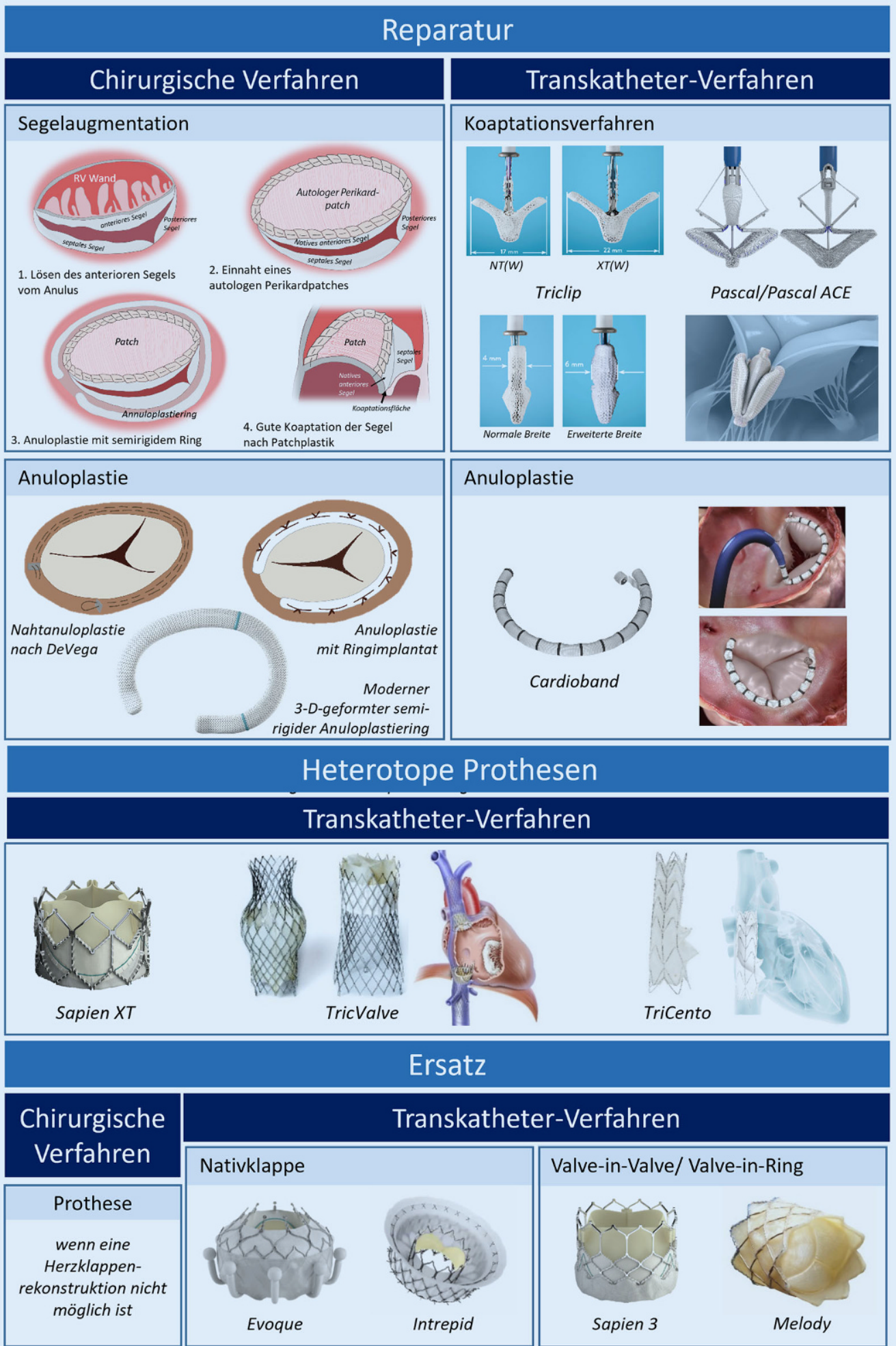

Abb. $3<$ Übersicht der chirurgischen und Transkatheterverfahren zur Behandlung der Trikuspidalklappeninsuffizienz (TI). (Mit freundl. Genehmigung von Abbott, Edwards Lifesciences Services, $\mathrm{P}+\mathrm{F}$ Products+Features $\mathrm{GmbH}$, Medira $\mathrm{GmbH}$, Medtronic) 
Tab. 1 Anspruch an chirurgische und Transkatheter-TK(Trikuspidalklappe)-Therapien

\begin{tabular}{|c|c|}
\hline Chirurgische TK-Therapie & Transkatheter-TK-Therapie \\
\hline \multicolumn{2}{|l|}{ Geringstmögliche Invasivität } \\
\hline Laterale Minithorakotomie & Femoraler venöser Zugangsweg \\
\hline "Beating heart" & \multirow[t]{3}{*}{ Frühe Entlassung (nach 2 Tagen bei TEER) } \\
\hline Frühe Extubation im Operationssaal & \\
\hline Frühe Mobilisation & \\
\hline \multicolumn{2}{|l|}{ Keine/geringe Komplikationen } \\
\hline Keine residuelle Insuffizienz & $\begin{array}{l}\text { Keine (Ersatz) oder nur geringfügige (Rekonstruktion) } \\
\text { residuelle Insuffizienz }\end{array}$ \\
\hline \multirow[t]{2}{*}{ Geringe operative Komplikationen } & Kein Gradient \\
\hline & Keine Zugangswegkomplikationen \\
\hline \multicolumn{2}{|l|}{ Optimales Outcome } \\
\hline Keine rezidivierende Insuffizienz & Keine rezidivierende Insuffizienz \\
\hline \multirow{2}{*}{$\begin{array}{l}\text { Ringdesign, das spätere Valve-in- } \\
\text { Valve-Prozedur ermöglicht }\end{array}$} & Positives Remodeling bei hoher Sicherheit \\
\hline & Symptom- und Überlebensverbesserung \\
\hline
\end{tabular}

und G4) und CLASP EFS sowie die CLASP ॥ TR (PASCAL und PASCAL Ace) zeigen vielversprechende Erfolge in der signifikanten Reduktion bei moderaten und schwergradigen Insuffizienzen, jedoch ist der Therapieansatz bei Vorliegen schwerster Insuffizienzen (TI Grad 4/5 oder 5/5) und größerer Koaptationslücken zunehmend weniger effektiv [5, 6]. Bei sehr großen Koaptationsdefekten $(\geq 8,4 \mathrm{~mm}$ ) oder bei einem sehr starkem Tethering ist dieses Verfahren technisch nur eingeschränkt oder nicht geeignet [7].

Ein weiteres Reparaturverfahren ist die dem chirurgischen Vorgehen angelehnte interventionelle Anuloplastie (Cardioband, Edwards Lifesciences, Irvine, (A, USA), die bei reiner Anulusdilatation solitär oder bei kombinierten Pathologien als COMBO- oder Hybrideingriff mit TEER durchgeführt werden kann. Limitationen dieses Verfahrens sind die Prozedurdauer und der Kontrastmitteleinsatz bei CT und die prozeduralen wiederholten Darstellungen der benachbarten RCA zur Vermeidung von peri- und postprozeduralen Ischämien.

Die Revolution: TranskatheterTrikuspidalklappenersatz - Wo liegen die Herausforderungen?

Neue Hoffnung für schwerkranke Patienten mit hochgradiger $\mathrm{TI}$ und einem starken Tethering der Segel mit relevanten Erfolgseinschränkungen für TK-
Die besonderen Vorteile der TK, die zu einer Entwicklungspräferenz interventioneller Klappenersatzsysteme führen, sind:

- fehlende Obstruktionsmöglichkeit des rechtsventrikulären Ausflusstraktes,

- einfacher großvolumiger Zugang über Femoralvenen, V. cava und rechtes Atrium,

- niedrige Druckverhältnisse, die das Risiko der Embolisation oder Migration der Klappe verringern,

- geringe Hämolysegefahr bei paravalvulären Lecks,

- Platz für Klappengrößen jenseits von $29 \mathrm{~mm}$, die niedrige Gradienten ermöglichen und auch eine Valve-inValve-Folgebehandlung begünstigen.

Transkatheter-Trikuspidal-

klappenersatz - innovative

Devicesysteme

Entwicklung der Transkatheter-TK-Ersatzsysteme. Im Vergleich zur etablierten TAVI ("transcatheter aortic valve implantation $^{\prime \prime}$ ) in Aortenklappenposition erfordern die variablen anatomischen Verhältnisse der TK eine deutlich aufwendigere Eingriffsplanung.

Die besonderen technischen Herausforderungen der TK sind:

- größte Herzklappe mit hochvariablen Ringdiametern von 30-65 mm,

- sehr komplexe 3-D-Anatomie,

- asymmetrischer, variabel sattelförmiger Anulus mit ellipsoider Ringstruktur,

- das Gewebe des TK-Anulus ist im Vergleich zu den linksseitigen Klappen weniger stark fibrös und steif und bietet somit weniger Halt bei der Implantation,

- starke Deformierungsdynamik des Anulus mit hoher Größenvarianz in Systole und Diastole,

- keine Verkalkung im Bereich der Prothesenlandezone,

- anteroseptale Beziehung des TKAnulus und Koch-Dreieck mit dem AV-Knoten, die zu hämodynamisch relevanten Pausen bis AV-Block III führen kann,

- langsamer Blutfluss und niedriger Segelöffnungs- und -schließungsdruck mit Gefahr der Thrombosierung.
Von den bisher entwickelten Transkatheter-TK-Ersatz-Systemen haben es nur wenige $z u$ "First-in-man"-Implantationen geschafft. Bei allen Systemen handelt es sich um selbstexpandierende Prothesen, die sich aber hinsichtlich Form und Verankerungsmechanismus relevant unterscheiden.

Die erste größere Compassionate-useSerie von 30 Patienten des GATE-Systems (NaviGate Cardiac Structures, Inc., Lake Forest, CA, USA) wurde 2020 berichtet [2]. Das Implantationssystem wird über einen 42 French (F) großen transjugulären oder meist direkten rechtsatrialen Zugang implantiert. Im Vergleich dazu benötigt eine TEER einen 22- bis 25-F- und eine TAVI nur einen 14- bis 16-F-Zugang. Wenn auch etwas weniger invasiv als ein traditionell chirurgischer Ansatz, zeigten sich aufgrund der großen Schleusengröße und des rigiden Steuerungssystems relevante Blutungskomplikationen ( $87 \%$ technischer Erfolg, 83\% rechtsatrialer Zugang, $13 \%$ Blutungskomplikationen, 7\% intraprozedurale chirurgische Konversion, $10 \%$ intrahospitale Mortalität).

Für das neuere EVOQUE Tricuspid Valve Replacement-System (Edwards Lifesciences, Irvine, CA, USA) wurde kürzlich die erste weltweite Compassionate-useSerie von 25 Patienten berichtet [1]. Dieses System wird im Vergleich zum GATESystem über einen transvenösen, trans- 

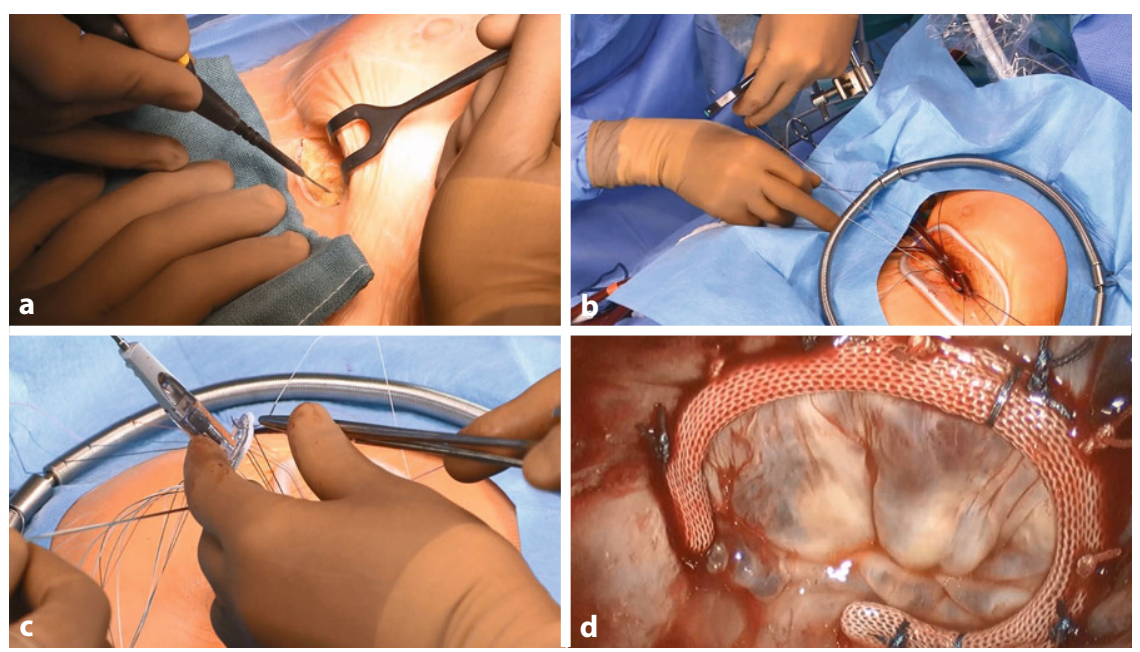

Abb. 4 A Minimal-invasive endoskopische TK(Trikuspidalklappen)-Rekonstruktion am schlagenden Herzen. a Anterolaterale Minithorakotomie. b Situs nach Einbringen des Weichgeweberetraktors und der 3-D-Kamera. c Einnaht des Anuloplastieringes. d Gute Koaptation der Segel nach Anuloplastie

femoralen und signifikant kleineren 28F-Zugang appliziert. Die Prothese (GröBen 44,48 und $52 \mathrm{~mm}$ ) verfügt über 9 Anker, die sich von ventrikulär in den Klappensegeln fixieren. Für das berichtete Hochrisikokollektiv (mittlerer STS-Score $9 \%)$ konnte eine hohe technische Erfolgsrate mit signifikanter klinischer Besserung gezeigt werden. Es gab keinen Fall einer intraprozeduralen chirurgischen Konversion, die 30-Tage-Sterblichkeit lag bei $0 \%$, und die Komplikationsrate war gering $(8 \%$ Blutungen, 12\% Schrittmacherpflicht, 4\% Dialysepflicht). Eine randomisierte Multicenterstudie (TRISCEND II, NCT04482062) zur Evaluation der Sicherheit und Performance des EVOQUE-Systems mit geplanten 775 Patienten wurde kürzlich gestartet. Sie stellt die erste randomisierte Klappenersatzuntersuchung gegen eine medikamentöse Therapie dar.

Das Intrepid-System (Medtronic, Minneapolis, MN, USA) ist für den Transkatheter-Mitral- und TK-Ersatz geeignet. Die Prothese besteht aus einem äußeren, an den Anulus formanpassungsfähigen Stent $(42$, 48 und $52 \mathrm{~mm}$ ), in den ein innerer zirkulärer Stent aufgehängt ist und die trikuspide 27mm-Klappe umschließt. Die Implantation erfolgt über einen transvenösen, transfemoralen 35-F-Zugang. Eine klinische Studie mit geplanten 15 Patienten wurde im Oktober 2020 gestartet (TTVR Early Feasibility Study, NCT04433065). Die neueste Version des Zuführungssystems konnte auf $29 \mathrm{~F}$ transvenös verkleinert werden, was den Leistenzugang venös noch attraktiver macht.

Neben den genannten Prothesen sind noch weitere Modelle in der Entwicklung (u.a. Topaz, TRiCares GmbH, Aschheim, Deutschland, LuX-Valve, Jenscare Biotechnology, Ningbo, China, VDYNEe, VDYNE LCC, Maple Grove, MN, USA, Trisol, Trisol Medical, Yokneam, Israel).

Bei an der TK voroperierten Patienten kann eine ballonexpandierende Prothese (Edwards Sapien 3, Edwards Lifesciences, Irvine, CA, USA oder Medtronic Melody, Medtronic, Minneapolis, MN, USA) in die vorimplantierte chirurgische Klappenprothese oder den Ring eingebracht werden.

Neben dem orthotopen TranskatheterTK-Ersatz stellt die heterotope Implantation von Klappenprothesen in die Hohlvenen (Sapien, Edwards Lifesciences, Irvine, CA, USA, TricValve, P\&F Products and Features $\mathrm{GmbH}$, Wien, Österreich, Tricento, Medira, Balingen, Deutschland) einen anderen Therapieansatz dar. Die eigentliche TK-Pathologie wird aber hierbei nicht adressiert, und das Verfahren dient v. a. der palliativen Symptomreduktion und Druckminderung im venös abdominellen und Beingefäßbett.

\section{Fazit für die Praxis}

- Neben dem Schweregrad der Trikuspidalklappeninsuffizienz (TI) müssen die RV(rechter Ventrikel)-Funktion und der Lungendruck/-widerstand bei jeder Vorstellung mit beurteilt werden.
- Entscheidend sind der Progress der Erkrankung und somit der Zeitpunkt der Umstellung einer medikamentösen auf eine interventionelle Therapie: Patienten müssen rechtzeitig in einem spezialisierten Zentrum für die weitere Therapieevaluation vorgestellt werden, bevor es zur irreversiblen RV-Schädigung kommt.

- Neue Therapieoptionen (minimal-invasive Operation, Transkatheter-TK[Trikuspidalklappe]-Reparatur/-Ersatz) erlauben in naher Zukunft einen patientenindividualisierten Therapieansatz mit dem Ziel einer klinisch symptomatischen und auch prognostischen Verbesserung.

- Die stetige Verbesserung der multimodalen Bildgebungstools trägt entscheidend zu einem optimalen Prozedurergebnis bei.

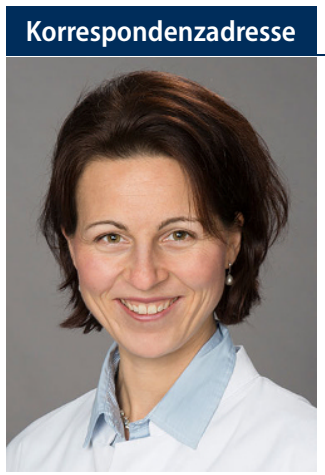

PD Dr. Michaela M. Hell

Zentrum für Kardiologie, Kardiologie 1, Universitätsmedizin Mainz

Langenbeckstr. 1, 55131 Mainz, Deutschland michaela.hell@unimedizin-mainz.de

Funding. Open Access funding enabled and organized by Projekt DEAL.

\section{Einhaltung ethischer Richtlinien}

Interessenkonflikt. R.S. von Bardeleben ist unbezahlter Principal Investigator und Mitglied von ECA für internationale Klappenstudien von Abbott, Edwards Lifesciences. Er erhält Vortragshonorare und ist im Advisory Board für Klappenentwicklungen der genannten Firmen. M.M. Hell, H. Treede und T. Münzel geben an, dass kein Interessenkonflikt besteht.

Für diesen Beitrag wurden von den Autoren keine Studien an Menschen oder Tieren durchgeführt. Für die aufgeführten Studien gelten die jeweils dort angegebenen ethischen Richtlinien.

Open Access. Dieser Artikel wird unter der Creative Commons Namensnennung 4.0 International Lizenz veröffentlicht, welche die Nutzung, Vervielfältigung, Bearbeitung, Verbreitung und Wiedergabe in jeglichem Medium und Format erlaubt, sofern Sie den/die ursprünglichen Autor(en) und die Quelle ordnungsgemäß nennen, einen Link zur Creative Commons Lizenz 
beifügen und angeben, ob Änderungen vorgenommen wurden.

Die in diesem Artikel enthaltenen Bilder und sonstiges Drittmaterial unterliegen ebenfalls der genannten Creative Commons Lizenz, sofern sich aus der Abbildungslegende nichts anderes ergibt. Sofern das betreffende Material nicht unter der genannten Creative Commons Lizenz steht und die betreffende Handlung nicht nach gesetzlichen Vorschriften erlaubt ist, ist für die oben aufgeführten Weiterverwendungen des Materials die Einwilligung des jeweiligen Rechteinhabers einzuholen.

Weitere Details zur Lizenz entnehmen Sie bitte der Lizenzinformation auf http://creativecommons.org/ licenses/by/4.0/deed.de.

\section{Literatur}

1. Fam NP, von Bardeleben RS, Hensey $M$ et al (2021) Transfemoral transcatheter tricuspid valve replacement with the EVOQUE system: a multicenter, observational, first-in-human experience. JACC Cardiovasc Interv 14:501-511

2. Hahn RT, Kodali S, Fam N et al (2020) Early multinational experience of transcatheter tricuspid valve replacement for treating severe tricuspid regurgitation. JACC Cardiovasc Interv 13:2482-2493

3. Hahn RT, Zamorano JL (2017) The need for a new tricuspid regurgitation grading scheme. Eur Heart JCardiovasc Imaging 18:1342-1343

4. Hell MM, Emrich T, Kreidel F et al (2021) Computed tomography imaging needs for novel transcatheter tricuspid valve repair and replacement therapies. Eur Heart J Cardiovasc Imaging 22:601-610

5. Kitamura M, Fam NP, Braun D et al (2021) 12-Month outcomes of transcatheter tricuspid valve repair with the PASCAL system for severe tricuspid regurgitation. Catheter Cardiovasc Interv 97:1281-1289

6. Lurz P, von Bardeleben RS, Weber M et al (2021) Transcatheter edge-to-edge repair for treatment of tricuspid regurgitation. J Am Coll Cardiol 77:229-239

7. Ruf TF, Hahn RT, Kreidel F et al (2021) Short-term clinical outcomes of transcatheter tricuspid valve repair with the third-generation Mitraclip XTR system. JACC Cardiovasc Interv 14:1231-1240

8. Vahanian A, Beyersdorf F, Praz F et al (2021) 2021 ESC/EACTS guidelines for the management of valvular heart disease. Eur Heart J. https://doi.org/ 10.1093/eurheartj/ehab395

\section{Modern treatment options for tricuspid valve regurgitation. From guideline-conform surgical repair and interventional cusp repair strategies to transcatheter tricuspid valve replacement}

The lack of awareness of the clinical relevance of tricuspid valve regurgitation as well as a high intrahospital mortality until recently led to a late patient referral frequently already with irreversible right ventricular damage. Innovative treatment approaches, new imaging techniques and an improved hemodynamic understanding of the right heart have revolutionized tricuspid valve treatment in recent years. The current review discusses the importance of right heart function for tricuspid valve treatment and presents modern interventional transcatheter and minimally invasive surgical techniques. Furthermore, it provides an overview of the current development of innovative transcatheter tricuspid valve replacement systems.

\section{Keywords}

Transcatheter heart valve treatment · Minimally invasive surgery · Right ventricular function . Intrahospital mortality · Hemodynamics 